\title{
THE 2006 OUTBURST OF THE MAGNETAR CXOU J164710.2 - 455216
}

\author{
Peter M. Woods ${ }^{1,2,3}$, Victoria M. Kaspi ${ }^{4,5}$, Fotis P. GavriIL $^{6,7}$, and Carol Airhart $^{1}$ \\ ${ }^{1}$ Dynetics, Inc., 1000 Explorer Boulevard Huntsville, AL 35806, USA \\ ${ }^{2}$ National Space Science and Technology Center, 320 Sparkman Drive Huntsville, AL 35805, USA \\ ${ }^{3}$ Corvid Technologies, 689 Discovery Drive, Huntsville, AL 35806, USA; peter.woods@ corvidtec.com \\ ${ }^{4}$ Department of Physics, Rutherford Physics Building, McGill University, 3600 University Street, Montreal, QC H3A 2T8, Canada \\ ${ }^{5}$ Astronomy Department, California Institute of Technology, 1200 East California Boulevard, Pasadena, CA 91125, USA \\ ${ }^{6}$ NASA Goddard Space Flight Center, Astrophysics Science Division, Code 662, Greenbelt, MD 20771, USA \\ ${ }^{7}$ Center for Research and Exploration in Space Science and Technology, University of Maryland, Baltimore County, 1000 Hilltop Circle, Baltimore, MD 21250, USA \\ Received 2009 July 13; accepted 2010 October 19; published 2010 December 13
}

\begin{abstract}
We report on data obtained with the Chandra, XMM-Newton, Suzaku, and Swift X-ray observatories, following the 2006 outburst of the Anomalous X-ray Pulsar CXOU J164710.2-455216. Using a more complete and higher signal-to-noise data set, we find no evidence for the very large glitch and rapid exponential decay as was reported previously for this source. We set a $3 \sigma$ upper limit on any fractional frequency increase at the time of the outburst of $\Delta v / v<1.5 \times 10^{-5}$. Our timing analysis, based on the longest time baseline yet, yields a spin-down rate for the pulsar that implies a surface dipolar magnetic field of $\sim 9 \times 10^{13} \mathrm{G}$, although this could be biased high by possible recovery from an undetected glitch. We also present an analysis of the source flux and spectral evolution, and find no evidence for long-term spectral relaxation post-outburst as was previously reported.
\end{abstract}

Key words: pulsars: individual (CXOU J164710.2-455216) - stars: individual (CXOU J164710.2-455216) - stars: magnetars - stars: neutron

\section{INTRODUCTION}

Of the various manifestations of isolated neutron stars, anomalous X-ray pulsars (AXPs), and soft gamma repeaters (SGRs) are the most dynamic members within the class (see Woods \& Thompson 2006 and Mereghetti 2008 for reviews). The extraordinary changes they undergo in radiative output are believed to be driven by the strong, evolving magnetic field which powers their bright X-ray emission (Thompson \& Duncan 1995, 1996; Thompson et al. 2002). Now generally recognized as magnetars, AXPs and SGRs are observed to have spin periods within a narrow range (2-12 s), possess rapid spin-down rates indicative of their strong surface dipolar field strengths $\left(10^{14}-10^{15} \mathrm{G}\right)$, usually maintain X-ray luminosities of $10^{33}-10^{36} \mathrm{erg} \mathrm{s}^{-1}$ though often with significant variability, and at times emit bright, often super-Eddington bursts of X-rays and gamma-rays. These sudden bursts from magnetars are often clustered in events referred to as "outbursts."

Scores of outbursts have been observed in SGRs since their discovery in 1979 (e.g., Gogus et al. 2010). Only since the discovery of pulsating X-ray counterparts in 1998 (Kouveliotou et al. 1998), however, have searches for correlated spectral and temporal changes been possible. The measured impact of SGR outbursts on the spectral and temporal properties of the persistent X-ray emission has been mixed. Extremely energetic individual SGR bursts or flares have triggered correlated spectral (e.g., Woods et al. 1999) and temporal variability (e.g., Israel et al. 2005). In general, however, SGR outbursts are not typically accompanied by spectral/temporal variability beyond the volatility which is seen in quiescence (Woods et al. 2007).

The first outburst from an AXP was not observed until 2002, when more than 80 individual bursts were recorded during a single $3 \mathrm{hr}$ observation of 1E 2259+586 (Kaspi et al. 2003). These bursts were accompanied by a sudden spin-up "glitch" of fractional spin-frequency change $\sim 4 \times 10^{-6}$. Other examples of radiative outbursts accompanied by glitches have been seen in AXPs 1E 1048.1-5937 (Dib et al. 2009) and 4U $0142+61$ (Gavriil et al. 2009). Such outbursts are believed to be caused by a fracturing of the neutron star crust, a result of internal magnetic stresses, and include the external surface and magnetospheric disturbances that follow. The spin-up glitch and subsequent relaxation can be interpreted in terms of angular momentum transfer from an initially more rapidly spinning crustal superfluid to the crust, as mediated by unpinning and later re-pinning of the superfluid angular momentum vortex lines.

The 2003 outburst of XTE J1810-197 (Ibrahim et al. 2004) provided yet another opportunity to study an AXP outburst. In this case, the AXP brightened by a factor $\sim 300$ directly post-outburst, as compared to the factor $\sim 20$ flux increase observed for 1E 2259+586. Not having been monitored prior to the outburst, it is not known if a spin-up glitch accompanied this event.

Several glitches in AXPs have been detected though with no obvious radiative outburst. These include glitches having comparable size to that seen in $1 \mathrm{E} 2259+586$ at the time of its outburst (e.g., Kaspi \& Gavriil 2003; Dall'Osso et al. 2003; Dib et al. 2008). A correlation between radiative behavior and glitch activity has been claimed for 1RXS J170849.0-400910 (Rea et al. 2005; Campana et al. 2007; Israel et al. 2007b); however, Dib et al. (2008) argue that the evidence for a correlation in this source thus far is marginal. Some of the larger AXP glitches have recoveries that are unusual when compared with those of rotation-powered pulsars, hinting at possible structural differences with magnetars (Woods et al. 2004; Dib et al. 2008; Gavriil et al. 2009; Livingstone et al. 2010). Establishing the existence and properties of AXP glitches, particularly for those for which there are accompanying observable radiative changes, offers a view of the impact of the internal event on the surface and immediate stellar surroundings, hence is important.

In 2006, another AXP outburst was detected, this time from CXOU J164710.2-455216. This outburst was signaled 
by a bright X-ray burst detected with the Swift observatory (Krimm et al. 2006) on 2006 September 21. The AXP CXOU J164710.2-455216 (CXO J1647 hereafter) is a $10.6 \mathrm{~s}$ X-ray pulsar located in the young cluster of massive stars, Westerlund 1 (Muno et al. 2006b). Several Target-of-Opportunity (ToO) observations with multiple X-ray telescopes were performed following the burst detection. Early Swift XRT (X-ray Telescope; Campana \& Israel 2006) and XMM-Newton (Muno et al. 2006a) ToO observations showed that the flux from the AXP increased by a factor $\sim 100$ following the outburst-similar in magnitude to the outburst from XTE J1810-197. A fortuitous $X M M-N e w t o n$ observation performed just four days prior to the CXO J1647 outburst provided a baseline for comparison. Muno et al. (2007) showed that the spectrum of CXO J1647 hardened significantly when the flux increased and that the pulse profile changed dramatically from a simple near-sinusoidal morphology to a complex profile with three distinct peaks per cycle. They argued that currents in the magnetosphere of CXO J1647 were excited during this event, causing the enhanced radiative output. These same currents could also explain the change in pulse profile by altering the opacity of the magnetosphere. Muno et al. (2007) predict that as these currents relax and decay, the pulse profile will return to its pre-outburst morphology.

From our first four Chandra ToO observations obtained in an interval of 6-37 days after the burst, we made a preliminary measurement of the spin-down rate of CXO J1647 (Woods et al. 2006). From a separate phase-coherent timing analysis of the XMM-Newton and Swift data on CXO J1647, Israel et al. (2007a) claimed evidence for an enormous glitch of magnitude $\Delta v / v \simeq 6 \times 10^{-5}$ at some point between the two XMM-Newton observations bracketing the outburst. If correct, this glitch would represent the largest fractional frequency change yet seen for any neutron star, hence would be of great importance. The frequency jump caused by the putative glitch was reported by Israel et al. (2007a) to have decayed exponentially with an $e$-folding time of $\sim 1.5$ days, remarkably rapid even by AXP standards.

Here, we report on a sequence of five Chandra ToO observations of CXO J1647 from six days following the outburst to 2007 February 2. Also included in our analysis are archival data from three XMM-Newton observations, one observation with Suzaku and 15 observations with the Swift XRT. Combining these data, we report on the flux decay, spectral evolution, pulse morphology changes, and pulse timing of CXO J1647 during the first several months of its post-outburst recovery. As we show in this paper, our analysis reveals no evidence for the previously claimed glitch in CXO J1647.

\section{X-RAY OBSERVATIONS}

As part of our ongoing Chandra ToO program for AXPs, we observed CXO J1647 on five separate occasions with the Chandra ACIS detector (Weisskopf et al. 2000) between 2006 September 28 and 2007 February 02. All observations utilized the ACIS-S3 chip operated in Continuous Clocking (CC) mode. This detector mode provides only a one-dimensional image, but very fine time resolution $(2.85 \mathrm{~ms})$ and thus a larger dynamic range of measurable source intensities. In Table 1, we list relevant details of the Chandra observations including the source exposure times, time of the observations, detector mode, and observation database reference numbers.

By coincidence, CXOU J164710.2-455216 was observed just four days prior to the outburst on 2006 September 21 by XMM-Newton (Muno et al. 2007). We report on this observation and on two additional observations obtained on 2006 September
22 and on 2007 February 17 (see Table 1). In particular, we report on results from the PN detector which was operated in Full Window (FW) mode for the first two pointings and Large Window (LW) mode during the 2007 observation (Jansen et al. 2001). The PN-FW mode offers two-dimensional imaging and $73 \mathrm{~ms}$ time resolution. The LW mode also has two-dimensional imaging, but with finer time resolution $(48 \mathrm{~ms})$ and thus a larger dynamic range. The details of these observations are contained in Table 1.

Following the announcement of burst activity from CXO J1647, Suzaku also declared a ToO and observed the AXP within 2.5 days of the Swift burst detection (Table 1). Here, we utilize data recorded by the four XIS detectors onboard Suzaku (Koyama et al. 2007) operated in $1 / 8$ window mode. The XIS detectors time tag photons to within $1.0 \mathrm{~s}$ and provide twodimensional spatial information. The point-spread functions of the four Suzaku XRTs matched to the XIS detectors have halfpower diameters $\sim 2^{\prime}$.

Finally, the Swift XRT detector (Burrows et al. 2005) observed CXO J1647 15 times between 2006 September 17 and 2007 April 18 (Table 1). During these observations, the detector operated in either Photon Counting (PC) or Windowed Timing (WT) mode depending upon the source brightness and pre-compiled commands to the spacecraft. In WT mode, the XRT detector records a one-dimensional image with fine time resolution $(2.2 \mathrm{~ms})$, analogous to Chandra CC mode. In PC mode, two-dimensional information is recorded, but with very coarse time resolution $(2.5 \mathrm{~s})$ and a much more limited dynamic intensity range.

\section{TEMPORAL ANALYSIS}

For each of the five Chandra ACIS observations of CXO J1647, we started with the standard level 2 filtered event list, using CIAO v4.0 ${ }^{8}$. First, we found the centroid for the peak of the one-dimensional image from each $\mathrm{CC}$-mode observation and selected counts within 4 pixels of the centroid (i.e., $\pm 2^{\prime \prime}$ ). We further selected counts with measured energies between 0.5 and $7.0 \mathrm{keV}$ and constructed light curves with $0.5 \mathrm{~s}$ resolution. No bursts were observed in any of the Chandra observations of CXO J1647.

Next, we converted the photon arrival times to the solar system barycenter using the CIAO tool axbary and a J2000 source position of $\alpha=16^{\mathrm{h}} 47^{\mathrm{m}} 10^{\mathrm{s}} .2$ and $\delta=-45^{\circ} 52^{\prime} 16^{\prime \prime} .90$ (Muno et al. 2006). A simple fast Fourier transform (FFT) of each light curve revealed a clear pulsed signal at the spin frequency of CXO J1647. The Fourier spectrum showed strong harmonic content up to the third harmonic above the fundamental frequency.

Starting from the Observations Data Files, we constructed filtered event lists for the two post-burst XMM-Newton observations using the epchain tool provided within the XMM-Newton Science Analysis Software (XMMSAS) v7.0.0 package. In the filtering process, we followed standard filtering procedures for PN data and retained counts with patterns 0 through 12 . Next, we extracted source and background event lists from circular regions of $35^{\prime \prime}$ and $50^{\prime \prime}$, respectively. The background regions were selected from the same CCD that contained the source. We further excluded time periods when a flare was clearly visible in the background light curve and the average count rate in the source region increased by more than $10 \%$. We next filtered on energy retaining counts between 0.5 and $7.0 \mathrm{keV}$. Finally, the

\footnotetext{
8 http://cxc.harvard.edu/ciao/
} 
Table 1

X-ray Observation Log for CXOU J164710.2-455216 Between 2006 September and 2007 February

\begin{tabular}{|c|c|c|c|c|c|c|}
\hline Name & Observatory & Detector & Obsid & $\begin{array}{c}\text { Time Relative to } \\
\text { Swift Burst } \\
\text { (days) }\end{array}$ & $\begin{array}{c}\text { Date }^{\mathrm{a}} \\
\text { (MJD TDB) }\end{array}$ & $\begin{array}{c}\text { Exposure } \\
(\mathrm{ks})\end{array}$ \\
\hline Obs 1 & $X M M$ & EPN & 0404340101 & -4.01 & 53995.06 & 40.7 \\
\hline Obs 2 & Swift & XRT & 00030806001 & 0.79 & 53999.85 & 1.9 \\
\hline Obs3 & Swift & XRT & 00030806002 & 1.55 & 54000.62 & 0.8 \\
\hline Obs4 & $X M M$ & EPN & 0311792001 & 1.64 & 54000.70 & 26.8 \\
\hline Obs5 & Swift & XRT & 00030806003 & 2.01 & 54001.07 & 4.9 \\
\hline Obs6 & Suzaku & XIS & 901002010 & 2.62 & 54001.69 & 154.8 \\
\hline Obs7 & Chandra & ACIS & 6724 & 6.31 & 54005.38 & 15.2 \\
\hline Obs8 & Chandra & ACIS & 6725 & 11.04 & 54010.11 & 20.2 \\
\hline Obs9 & Swift & XRT & 00030806006 & 11.57 & 54010.64 & 2.0 \\
\hline Obs 10 & Swift & XRT & 00030806007 & 12.49 & 54011.56 & 2.0 \\
\hline Obs11 & Swift & XRT & 00030806008 & 14.99 & 54014.05 & 2.2 \\
\hline Obs 12 & Chandra & ACIS & 6726 & 18.36 & 54017.42 & 25.2 \\
\hline Obs 13 & Swift & XRT & 00030806009 & 18.78 & 54017.85 & 3.5 \\
\hline Obs14 & Swift & XRT & 00030806010 & 18.99 & 54018.05 & 2.8 \\
\hline Obs 15 & Swift & XRT & 00030806011 & 24.32 & 54023.38 & 5.6 \\
\hline Obs 16 & Swift & XRT & 00030806012 & 30.17 & 54029.24 & 5.5 \\
\hline Obs17 & Swift & XRT & 00030806013 & 36.69 & 54035.76 & 2.8 \\
\hline Obs 18 & Chandra & ACIS & 8455 & 37.32 & 54036.39 & 15.2 \\
\hline Obs19 & Swift & XRT & 00030806014 & 120.15 & 54119.21 & 2.1 \\
\hline Obs 20 & Swift & XRT & 00030806015 & 123.06 & 54122.13 & 3.8 \\
\hline Obs 21 & Chandra & ACIS & 8506 & 134.86 & 54133.92 & 20.2 \\
\hline Obs 22 & $X M M$ & $\mathrm{EPN}$ & 0410580601 & 149.41 & 54148.47 & 17.3 \\
\hline Obs 23 & Swift & XRT & 00030806016 & 208.32 & 54207.38 & 4.3 \\
\hline Obs24 & Swift & XRT & 00030806017 & 209.16 & 54208.23 & 2.2 \\
\hline
\end{tabular}

Note. ${ }^{a}$ Midpoint of observation.

photon time tags were corrected to the Solar system barycenter using the XMMSAS tool barycen.

For the one Suzaku observation, we utilized the four XIS cleaned event lists produced by the standard pipeline analysis (v1.2.2.3). Following the recipe outlined in the Suzaku ABC Guide ${ }^{9}$, we constructed a circular source region ( $1^{\prime}$ radius) centered on the CXO J1647 position. Note that a larger region that encompassed more of the source flux could not be used because of the $1 / 8$ window observing mode of the XIS detectors for this observation. The spatial filtering was performed using the tool Xselect and the four resulting source event lists were merged into a single source event list using a custom Interactive Data Language (IDL) procedure. Energy filtering removed all counts outside of the 0.5 to $7.0 \mathrm{keV}$ range. Next, we corrected a systematic error ${ }^{10}$ in the photon arrival times by adding $7.0 \mathrm{~s}$ to each time tag. Finally, the Suzaku HEASOFT tool aebarycen was used to convert the photon arrival times to the Solar system barycenter.

Fifteen Swift observations of CXO J1647 were processed using HEASOFT v6.3.1. The Perl script cxrtpipeline generated level 2 data for each data set. Source and background regions were defined for $\mathrm{PC}$ observations as circles with radii of $47^{\prime \prime}$ and $118^{\prime \prime}$, respectively, with the background region selected to be sufficiently distant from the source and other point sources. For WT observations, the source region was defined as a $40 \times 10$ pixel $\left(\sim 94^{\prime \prime} \times 23^{\prime \prime}\right)$ rectangle, oriented along the readout direction of the $\mathrm{CCD}$. Background regions of the same dimensions were defined on either side of the source along the readout direction. Source and background event lists were ex-

\footnotetext{
9 http://heasarc.gsfc.nasa.gov/docs/suzaku/analysis/abc/

10 http://www.astro.isas.jaxa.jp/suzaku/analysis/xis/timing/
}

tracted using Xselect with a photon energy selection window of 0.5 to $7.0 \mathrm{keV}$ and the time tags were then corrected to the solar system barycenter using barycorr.

\subsection{Pulse Ephemeris}

As discussed earlier, there is some disagreement regarding the spin evolution of CXO J1647 following the burst activity in 2006 September. Our initial report on a subset of the Chandra observations showed evidence for rapid spindown (Woods et al. 2006), but not for exponential glitch recovery as was claimed by Israel et al. (2006) based upon XMM-Newton and Swift data. A subsequent publication by the same group (Israel et al. 2007a) included some Chandra data and resulted in generally the same conclusion of a large amplitude glitch $\left(\Delta v / v \simeq 6 \times 10^{-5}\right)$ with a rapid exponential glitch recovery on a timescale of $\sim 1$ day. Here, we consider all data available from this time period in an effort to resolve this apparent discrepancy. Note that the discrepancy applies only to the first $\sim 1$ week post-burst due to the rapid decay of the exponential term in the Israel et al. phase model.

In our analysis, we have invoked the commonly used technique of phase-coherent timing (Woods et al. 2004). The data were split into discrete segments according to detector type and time such that there are no gaps in any one data segment larger than one day. Using these criteria, each Chandra, $X M M-N e w t o n$, and Suzaku observation was grouped into an individual data segment. Some Swift pointings were combined with adjacent observations due to their close proximity in time. The last two Swift pointings were excluded due to their relatively low signal-to-noise. In total, 16 unique data segments were assembled. 

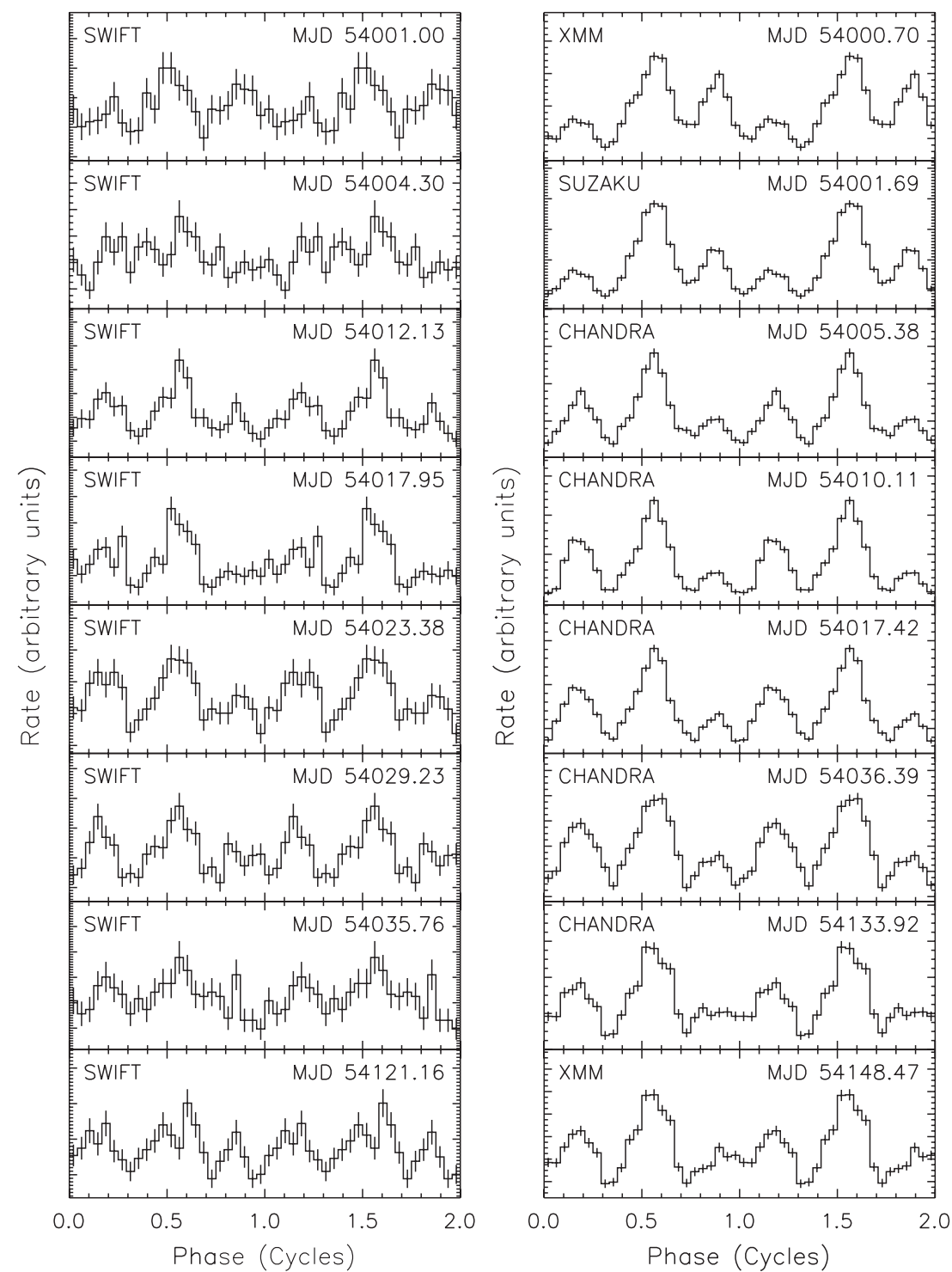

Figure 1. Left: Pulse profiles of CXOU J164710.2-455216 between 0.5 and $7.0 \mathrm{keV}$ from Swift data. Time progresses from top to bottom although not exactly at the same rate in each column. The total time durations are roughly the same. The profiles are phase aligned using our best-fit third-order polynomial ephemeris that includes no glitch. Note the difficulty in determining proper phasing, particularly at early times, from the Swift-observed pulse morphologies independently. Right: Pulse profiles of CXOU J164710.2-455216 between 0.5 and $7.0 \mathrm{keV}$ during the post-burst time period for data from Chandra, Suzaku, and XMM. Time progresses from top to bottom. The profiles are phase aligned using our best-fit 3rd order polynomial ephemeris that includes no glitch. Due to the different instruments used for this comparison, count rate units have been excluded. Note the gradual change in pulse morphology from the first pointing to the most recent.

Each data segment was folded using a pulse-phase model defined as a Taylor expansion of the phase $\phi(t)$ about a given epoch in time $t_{0}$, where $\phi(t)=\phi\left(t_{0}\right)+v\left(t-t_{0}\right)+\frac{1}{2} \dot{v}\left(t-t_{0}\right)^{2}+\ldots$. The pulse phase, frequency, and frequency derivative at time $t_{0}$ are given by $\phi, v$, and $\dot{v}$, respectively. Initially, we set these model parameters equal to the values determined by our earlier phase-coherent analysis of the Chandra data (Woods et al. 2006).

When constructing the folded pulse profiles, we chose 32 phase bins per cycle and selected counts within the energy range $0.5-7.0 \mathrm{keV}$ to provide good signal-to-noise and sufficient phase resolution for cross-correlation. Each of the 32 phase bins corresponds to $\sim 0.33 \mathrm{~s}$ in time. The majority of our data sets have time resolution much smaller than the size of our phase bins, thus we are justified in assigning all counts in a given detector accumulation interval to a single phase bin. The Swift XRT data accumulated in PC mode, on the other hand, have time resolution much coarser than our phase bins.
For this reason, we have "split" these counts across multiple phase bins when folding the data. This has the effect of smearing out features in the pulse profile as we are effectively convolving a rectangular function of phase width 0.33 cycles (the PC-mode time resolution divided by the pulse period) with the pulse profile. This smearing is mitigated to some extent by the fact that we are averaging over several hundred cycles at a time. Nevertheless, the distortion of the pulse profiles in the Swift data is not insignificant (see Figure 1, left).

Folded pulse profiles for each data segment were then crosscorrelated in the Fourier domain with a high signal-to-noise pulse template derived from the 2006 Chandra observations. The cross-correlation utilized amplitude and phase information from the fundamental plus the first three harmonics of the Fourier decomposition of the pulse profiles.

Visual inspection of the resulting initial phase residuals from a simple quadratic model (i.e., fitting for $\phi\left(t_{0}\right), v, \dot{v}$ ) showed a number of outliers within the first few days of the burst activity, 


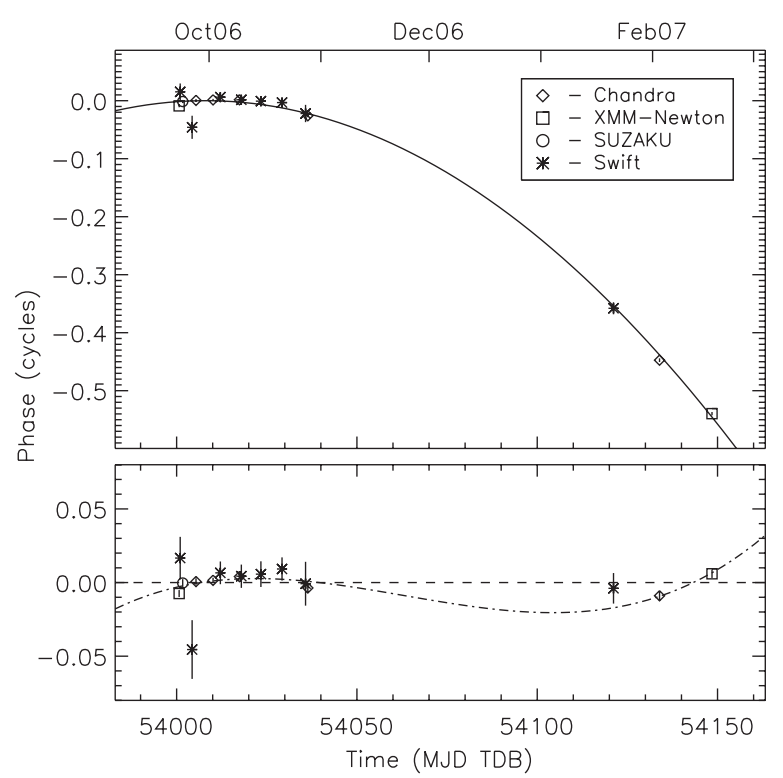

Figure 2. Pulse phases of CXOU J164710.2-455216 between 2006 September 23 and 2007 February 17. Top: pulse phases minus a linear phase model. The solid line indicates a quadratic fit to these phase measurements. Bottom: pulse phases minus a quadratic trend. The dash-dotted line indicates a cubic fit to the residuals.

but only, as expected, for the Swift XRT measurements. All other data points (in 2006) followed the quadratic trend. In fact, the small number of Swift outliers followed a similar quadratic trend, only this trend was offset in phase from the other model by $\sim 1 / 3$ cycles - the separation between the three peaks of the post-burst CXO J1647 pulse profile.

The Swift pulse profiles (Figure 1, left) have signal-to-noise ratio significantly less than those of the other pulse profiles (Figure 1, right). The pulsed signal was clearly visible in these data, but in many cases the distinction between the three peaks of the pulse profile was not clear. Due to the generally very short exposure times for the Swift XRT observations (see Table 1), the extensive usage of PC mode for these observations with coarse time resolution, and the intrinsic variability of the CXO J1647 pulse profile, we chose not to use these data to define our CXO J1647 pulse ephemeris.

Next, we fit a quadratic phase model to only the Chandra/ XMM-Newton/Suzaku phases to determine a new pulse ephemeris. We repeated this procedure folding in the 2007 data, and generated a new pulse template using the Chandra and $X M M-N e w t o n$ data. The best-fit quadratic model is presented in Table 2 and shown graphically in Figure 2 (top). The high $\chi^{2}$ (31.4 for 6 degrees of freedom) is indicative of a residual systematic trend. For this reason, we also tried including a cubic term in the phase fit and the residuals became more acceptable $\left(\chi^{2}=8.4\right.$ for 5 degrees of freedom). The resulting spin ephemeris for this cubic phase model is also given in Table 2 and Figure 2 (bottom).

For the quadratic fit, the measured $\dot{v}=-7.41(18) \times$ $10^{-15} \mathrm{~Hz} \mathrm{~s}^{-1}$, somewhat less than that measured by Woods et al. (2006), $-1.38(28) \times 10^{-14} \mathrm{~Hz} \mathrm{~s}^{-1}$. It is also less than that reported by Israel et al. (2007a). This is because of the longer baseline, and the systematic trend toward a smaller spin-down rate at later times. This $\dot{v}$ implies a magnetic field, calculated via $B=3.2 \times 10^{19}(P \dot{P})^{1 / 2} \mathrm{G}$, of $9.5 \times 10^{13} \mathrm{G}$. For the cubic fit, however, the measured instantaneous spin-down rate is even steeper, but is likely not stationary. The average $\dot{v}$ over the
Table 2

Spin Parameters for CXOU J164710.2-455216 from 2006 September 23 through 2007 February 17

\begin{tabular}{lc}
\hline \hline Start observing epoch (MJD) & 54000.692 \\
End observing epoch (MJD) & 54148.483 \\
Epoch (MJD TDB) & 54008.0000 \\
\hline Quadratic Fit: & \\
\hline Spin frequency ${ }^{\mathrm{a}}, v(\mathrm{~Hz})$ & $0.0942448774(11)$ \\
Spin frequency derivative, $\dot{v}\left(\mathrm{~Hz} \mathrm{~s}^{-1}\right)$ & $-7.4(2) \times 10^{-15}$ \\
\hline Cubic Fit: & \\
\hline Spin frequency, $v(\mathrm{~Hz})$ & $0.0942448813(14)$ \\
Spin frequency derivative, $\dot{v}\left(\mathrm{~Hz} \mathrm{~s}^{-1}\right)$ & $-1.14(9) \times 10^{-14}$ \\
Second spin frequency derivative, $\ddot{v}\left(\mathrm{~Hz} \mathrm{~s}^{-2}\right)$ & $8.5(18) \times 10^{-22}$ \\
\hline
\end{tabular}

Notes. ${ }^{a}$ Numbers in parentheses represent $1 \sigma$ uncertainties in the least significant digits quoted.

reported time span was $-6.5 \times 10^{-15} \mathrm{~Hz} \mathrm{~s}^{-1}$. If correct, this would imply a surface dipolar magnetic field of $B=8.9 \times 10^{13}$ $\mathrm{G}$. Thus, with the data reported here, we estimate the magnetic field strength at $\sim 9 \times 10^{13} \mathrm{G}$, somewhat less than reported by Woods et al. (2006) and Israel et al. (2007a).

The standard pulse-phase fitting technique assumes that features in the folded pulse profile remain stationary and do not drift in phase relative to the rotation of the star. A gradual, systematic drift of the orientation of the pulse maxima could mimic bona fide variations in the stellar rotation rate. For CXOU $\mathrm{J} 164710.2-455216$, this would require a systematic and selfconsistent shift in the three pulse maxima. Although this may explain some portion of the cubic term in the phase residuals, the dominant quadratic term $(\sim 0.5$ cycles $)$ and the $\sim 1 / 3$ cycles phase offsets cannot be accounted for by this effect as this would require a very contrived, unphysical shift in pulse phase.

Although the energy selection intervals $(0.5-7.0 \mathrm{keV})$ were the same for each pulse profile, the average photon energy for pulse profiles extracted from each instrument were not exactly the same due to slight differences in the effective area as a function of energy for the individual detectors. As there is no measurable, systematic phase offset observed between detectors in the pulse timing residuals other than those caused by pulse peak misidentification, the detector energy response differences must be minor relative to the intrinsic temporal and spectral (Israel et al. 2007a) variability of the CXOU J164710.2-455216 pulse profile.

Due to the extreme change in pulse profile from before the burst activity to following it, we could not phase connect to the 2007 September 17 XMM-Newton observation (Obs1). We measure a pulse frequency of $0.0942447(5) \mathrm{Hz}$ during this preburst observation, consistent with the values reported by Muno et al. (2006a) and Israel et al. (2007a). An extrapolation of our post-burst spin ephemeris (Table 2) to the time of the burst is consistent with the pre-burst spin frequency. Thus, we place a $3 \sigma$ upper limit on a glitch in CXO J1647 at the time of the burst activity at $\Delta v / v<1.5 \times 10^{-5}$. Our analysis of the complete sample of X-ray data during the time surrounding the 2006 burst activity shows no evidence for a glitch or an exponential glitch recovery of the magnitude and timescale as that reported by Israel et al. (2007a).

We believe that the discrepancy between our timing results and those reported by Israel et al. (2007a) amounts to cycle count ambiguities between observations immediately following the burst activity and misidentification of peaks in the three- 


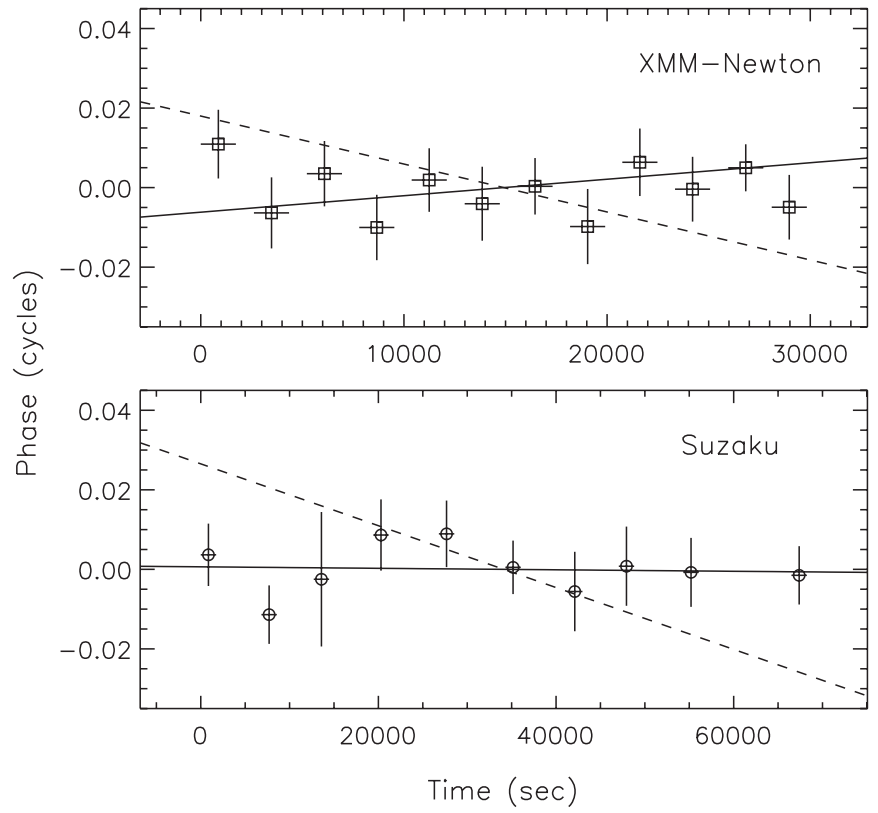

Figure 3. Pulse phases of CXOU J164710.2-455216 for the first XMM-Newton (top panel) and Suzaku (bottom panel) observations following the burst detection on 2006 September 21 . The solid line indicates the expected local frequency for the third-order polynomial model reported here (Table 2). The uncertainty in the frequencies for the polynomial model (i.e., slope of the line) are comparable to the thickness of the lines. Note that there is no significant difference in the instantaneous frequencies at these two epochs for the third-order and second-order polynomial models. The dashed line indicates the polynomial plus exponential model of Israel et al. (2007b). Frequency uncertainties for this model cannot be inferred directly from the fit results that have been reported.

peaked CXO J1647 post-burst pulse profile for some of the Swift data sets. Proving the latter point would require a direct comparison between our pulse profile correlation technique and the approach employed by Israel et al. The former issue can be more easily investigated by measuring frequencies during the observations in close proximity to the burst activity.

As the frequency error $(\delta \nu)$ in an individual observation is inversely proportional to its duration, $\delta v_{j} \propto T_{j}^{-1}$, the longer observations are more suitable for comparison of our polynomial model to the polynomial plus exponential model of Israel et al. In addition, those observations closest to the burst (i.e., "glitch") epoch are most constraining due to the rapid decay of the putative exponential component. For these reasons, we made our frequency comparisons using the first XMM-Newton (Obs4) and Suzaku (Obs6) post-burst observations.

Using the same phase-coherent timing approach as before, we divided the two observations into 12 and 10 segments, respectively. In order to limit the effect of pulse profile changes, we constructed pulse templates from the complete data set of each observation folded on the best-fit local frequency. Phase offsets were measured for each segment and a linear phase model was fit to the residuals to refine the pulse frequency. We repeated this procedure generating a new template profile and frequency measurement. The pulse phases for each data set are shown in Figure 3. Next, we computed the pulse frequencies at each of these epochs for the two models under consideration. The difference in frequency between the model values and the bestfit local frequency is shown graphically by the overplotted lines in Figure 3. Our third-order polynomial model is represented by the solid line and the Israel et al. model is indicated by the dashed line.
It is clear from Figure 3 that the frequencies predicted by our model are more consistent with the measured data. In particular, the $\chi^{2} /$ dof for the XMM-Newton and Suzaku data sets evaluated using our phase model are 9.4/10 and 5.1/8, respectively. On the other hand, the Israel et al. model returns much larger $\chi^{2} /$ dof of $29.2 / 10$ and $44.0 / 8$, respectively. The probabilities that one would obtain $\chi^{2}$ values this large by chance are $1 \times 10^{-3}$ and $6 \times$ $10^{-7}$, respectively. Thus, we conclude that the polynomial plus exponential glitch model of Israel et al. (2007a) is inconsistent with the data.

\section{SPECTRAL ANALYSIS}

Using the source and background regions defined in Section 3, X-ray pulse invariant spectra were constructed for each of the Chandra, XMM-Newton, and Suzaku observations. Due to the low signal-to-noise ratio of the Swift data, they were excluded from the spectral analysis. Response matrices were constructed using mkacisrmf from CIAO v4.1.2 and rmfgen from XMMSAX v7.1 for the Chandra and XMM-Newton data, respectively. Pre-computed $S u z a k u$ response matrices were utilized. The resulting energy spectra were grouped to ensure that at least 25 source counts were contained within each energy bin.

The spectra were simultaneously fit to a model defined by the sum of a blackbody and a power law modified by interstellar absorption. The spectra were fit using XSPEC v $11^{11}$ where only the interstellar absorption was forced to remain constant among data sets. We obtained a good fit to the data having a $\chi^{2}$ of 6208 for 6138 degrees of freedom. Fit-parameter uncertainties were estimated using the error command in XSPEC. Figure 4 shows the results of this fit, specifically the temporal evolution of the blackbody temperature, photon index ratio of 2-10 keV power-law flux to bolometric blackbody flux, and the total unabsorbed 2-10 keV flux. There is some indication of a slight drop in blackbody temperature at times greater than $\sim 2$ days following the outburst, however, the spectral fit results show no significant long-term variability in the spectral shape. The total unabsorbed 2-10 keV flux clearly decreases rapidly following the outburst. The flux decay is well described by a power law $\left(F=F_{0}\left(t-t_{0}\right)^{-\alpha}\right)$ with an index $\alpha=0.306 \pm 0.005$. In this fit, the epoch $\left(t_{0}\right)$ was fixed to the time of the peak of the outburst (53999.06554 MJD TDB).

\section{DISCUSSION}

Glitches in AXPs have now been shown to be generic to the class (e.g., Kaspi et al 2000; Kaspi \& Gavriil 2003; Dall'Osso et al. 2003), particularly at the times of large radiative outbursts (Kaspi et al. 2003; Dib et al. 2008; Gavriil et al. 2009). SGRs, on the other hand, have not shown similar timing anomalies, although the timing noise in these objects (e.g., Woods et al. 2000) makes detection of glitch activity more difficult. Given the closer connections CXOU J164710.2-455216 has with AXP-like magnetars, it would not be surprising if CXOU J164710.2-455216 suffered a glitch at the time of its 2006 outburst; indeed, given that every other well-observed AXP radiative outburst has included a timing anomaly, it might at first glance be surprising that our analysis of the existing data reveals none, especially given the previous claim by Israel et al. (2007a).

However, it is important to note that the $3 \sigma$ upper limit we place on the amplitude of any glitch that occurred is

\footnotetext{
11 http://heasarc.gsfc.nasa.gov/docs/xanadu/xspec/
} 

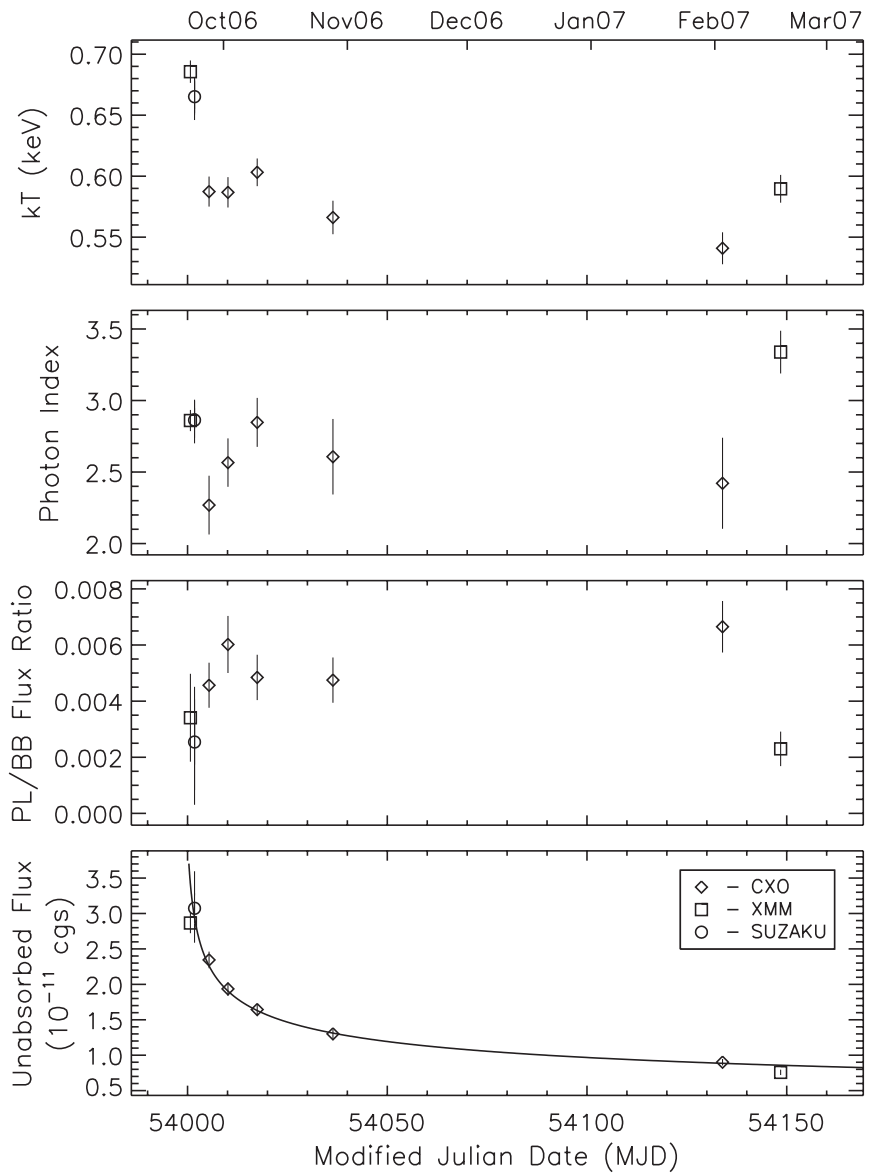

Figure 4. Spectral history of CXOU J164710.2-455216 between 2006 September 23 and 2007 February 17. Shown from top to bottom are the blackbody temperature, photon index, ratio of 2-10 keV power-law component flux to the bolometric blackbody flux, and total unabsorbed $2-10 \mathrm{keV}$ flux. Note that the blackbody component contributes the majority of the flux between 2 and $5 \mathrm{keV}$, whereas the power-law component dominates above and below this range. The small fraction in panel three arises from using the bolometric integration of the blackbody model and a finite integration for the power-law spectral model. The power-law fit $\left(F=F_{0}\left(t-t_{0}\right)^{-\alpha}\right)$ to the flux measurements is overplotted in the bottom panel. The best-fit power-law index was $-0.306 \pm 0.005$.

$\Delta v / v<1.5 \times 10^{-5}$, large by AXP (and indeed any) glitch standards. Only one AXP glitch seen thus far is higher (Dib et al. 2008). Thus, although our analysis rules out the extremely large glitch claimed by Israel et al. (2007a), it does not rule out a glitch having fractional amplitude similar to those seen in most AXP glitches. If CXOU J164710.2-455216 had been subject to phase-coherent timing prior to the event (impossible with, e.g., the Rossi X-ray Timing Explorer due to its pre-outburst faintness and a nearby, unrelated bright source), much smaller glitches would have been detectable.

The observed systematic deviation from simple spin-down, even in the relatively small interval covered by our observations (see Section 3), is interesting. The magnitude of $\dot{v}$ appears to have declined monotonically since the radiative outburst. This is suggestive of glitch recovery, even in the absence of direct evidence for a glitch. Strong glitch recoveries, with enhanced spin-down rates immediately post-glitch (with or without an accompanying radiative event) have been observed in other AXPs following large glitches (e.g., Kaspi et al. 2003; Kaspi \& Gavriil 2003; Dall'Osso et al. 2003). Their origin is unclear and may be signaling structural differences between magnetars and lower-magnetic field neutron stars (e.g., Dib et al. 2008). On the other hand, large variations in spin-down rate have been seen in other AXPs (e.g., Gavriil \& Kaspi 2004; Dib et al. 2009) in the absence of glitches, so we cannot conclude that glitch recovery is the origin of the $\dot{v}$ variation in CXOU J164710.2-455216.

If it were glitch recovery, however, and $\dot{v}$ is recovering to a smaller absolute value that is also its long-term average, then the true $\dot{v}$ could be even smaller than is reported here. Indeed, the instantaneous $\dot{v}$ at the end of the time span reported here (using the cubic phase model), if close to the long-term average, would imply a surface dipolar magnetic field strength of $3.7 \times 10^{13} \mathrm{G}$, somewhat low, but not lower than that of SGR $0418+5729$, which has $3 \sigma$ upper limit on its spin-down inferred $B$ of $3.2 \times 10^{13} \mathrm{G}$ (Esposito et al. 2010).

Israel et al. (2007a) reported a decay in the flux of CXOU J164710.2-455216 post-outburst that is well modeled by a power law of index $-0.28 \pm 0.05$, consistent with the index we observe, $-0.311 \pm 0.005$. As they remark, this is similar to what was observed for the flux decay of AXP 1E 2259+586 after its 2002 outburst (Kaspi et al. 2003; Woods et al. 2004; Zhu et al. 2008). However, Israel et al. (2007a) further report that when spectrally decomposing the emission, the power-law component decayed more rapidly than the blackbody component, with power-law indexes for these two components of $-0.38 \pm 0.11$ and $-0.14 \pm 0.10$, respectively. They argued that this implied that the cooling timescale for hypothesized surface hot spots was shorter than for relaxation of the region producing the power-law emission. However, as shown in Figure 4, when considering particularly the Chandra data, we find that the ratio of power-law- to blackbody-component flux remained relatively stable, or even increased slightly after $\sim 140$ days. We do not understand the origin of this observational discrepancy. The two decaying in concert is consistent with the picture of magnetospheric Compton scattering of enhanced surface thermal emission (Thompson et al. 2002; Zane et al. 2009) in the presence of decaying surface emission, with no change in the magnetospheric twist (e.g., Özel \& Güver 2007).

\section{CONCLUSIONS}

We have shown that there is no direct evidence to support the claim that AXP CXOU J164710.2-455216 in the massive star cluster Westerlund 1 exhibited a glitch at the time of its 2006 radiative outburst, and we set a $3 \sigma$ upper limit on the magnitude of any such glitch of $\Delta v / v<1.5 \times 10^{-5}$, which is larger than those seen in most other AXP radiative outbursts. We show that a previous claim by Israel et al. (2007a), that a far larger glitch occurred, was a result of misidentification of correct pulse phases in low signal-to-noise ratio data. We further show that the spin-down rate of CXOU J164710.2-455216 is lower than was measured immediately post-event, suggesting possible strong glitch recovery as has been seen in other AXPs, even in the absence of direct evidence for a glitch. The revised spin-down rate implies a surface dipolar magnetic field strength of $\sim 9 \times 10^{13} \mathrm{G}$, although strong glitch recovery could be biasing this result upward.

The authors wish to thank the referee for useful comments and the CXC help desk for guidance on the Chandra spectral analysis. P.M.W. is grateful for support from NASA through SAO grant GO7-8077A. V.M.K. thanks the California Institute of Technology for hospitality and acknowledges support from a Moore Scholarship, NSERC via a Discovery Grant, CIFAR, FQRNT, the Canada Research Chairs Program, and the Lorne Trottier Chair in Astrophysics and Cosmology. 


\section{REFERENCES}

Burrows, D. N., et al. 2005, Space Sci. Rev., 120, 165

Campana, S., \& Israel, G. L. 2006, ATel, 893

Campana, S., Rea, N., Israel, G. L., Turolla, R., \& Zane, S. 2007, A\&A, 463, 1047

Dall'Osso, S., Israel, G. L., Stella, L., Possenti, A., \& Perozzi, E. 2003, ApJ, 599,485

Dib, R., Gavriil, F. P., \& Kaspi, V. M. 2009, ApJ, 702, 614

Dib, R., Kaspi, V. M., \& Gavriil, F. P. 2008, ApJ, 673, 1044

Esposito, P., et al. 2010, MNRAS, 405, 1787

Gavriil, F. P., Dib‘, R., \& Kaspi, V. M. 2009, ApJ, submitted (arXiv:0905.1256)

Gogus, E., et al. 2010, ApJ, 718, 331

Ibrahim, A., et al. 2004, ApJ, 609, L21

Israel, G. L., Campana, S., Dall'Osso, S., Muno, M. P., Cummings, J., Perna, R., \& Stella, L. 2007a, ApJ, 664, 448

Israel, G. L., Dall'Osso, S., Campana, S., \& Muno, M. P. 2006, ATel, 932

Israel, G. L., Götz, D., Zane, S., Dall'Osso, S., Rea, N., \& Stella, L. 2007b, A\&A, 476, L9

Israel, G. L., et al. 2005, ApJ, 628, L53

Jansen, F., et al. 2001, A\&A, 365, L1

Kaspi, V. M., \& Gavriil, F. P. 2003, ApJ, 596, L71

Kaspi, V. M., Gavriil, F. P., Woods, P. M., Jensen, J. B., Roberts, M. S. E., \& Chakrabarty, D. 2003, ApJ, 588, L93

Kouveliotou, C., et al. 1998, Nature, 393, 235

Koyama, K., et al. 2007, PASJ, 59, S23

Krimm, H., Barthelmy, S., Campana, S., Cummings, J., Israel, G., Palmer, D., \& Parsons, A. 2006, ATel, 894
Livingstone, M. A., Kaspi, V. M., \& Gavriil, F. P. 2010, ApJ, 710, 1710

Mereghetti, S. 2008, A\&AR, 15, 225

Muno, M. P., Gaensler, B. M., Clark, J. S., de Grijs, R., Pooley, D., Stevens, I. R., \& Portegies Zwart, S. F. 2007, MNRAS, 378, L44

Muno, M. P., Gaensler, B. M., Clark, J. S., Portegies Zwart, S., Pooley, D., de Grijs, R., Stevens, I. R., \& Negueruela, I. 2006a, ATel, 902

Muno, M. P., et al. 2006b, ApJ, 636, L41

Özel, F., \& Güver, T. 2007, ApJ, 659, L141

Rea, N., Tiengo, A., Mereghetti, S., Israel, G. L., Zane, S., Turolla, R., \& Stella, L. 2005, ApJ, 627, L133

Thompson, C., \& Duncan, R. 1995, MNRAS, 275, 255

Thompson, C., \& Duncan, R. 1996, ApJ, 473, 322

Thompson, C., Lyutikov, M., \& Kulkarni, S. R. 2002, ApJ, 574, 332

Weisskopf, M. C., Tannanbaum, H. D., Van Speybroeck, L. P., \& O’Dell, S. L. 2000, SPIE, 4012, 2

Woods, P. M., Kaspi, V. M., \& Gavriil, F. P. 2006, ATel, 929

Woods, P. M., \& Thompson, C. 2006, in Compact Stellar X-ray Sources, ed. W. H. G. Lewin \& M. van der Klis (Cambridge: Cambridge Univ Press), 547

Woods, P. M., et al. 1999, ApJ, 524, L55

Woods, P. M., et al. 2000, ApJ, 535, L55

Woods, P. M., et al. 2004, ApJ, 605, 378

Woods, P. M., Kouveliotou, C., Finger, M. H., Gogus, E., Wilson, C. A., Patel, S. K., Hurley, K., \& Swank, J. H. 2007, ApJ, 654, 470

Zane, S., Rea, N., Turolla, R., \& Nobili, L. 2009, MNRAS, 398, 1403

Zhu, W., Kaspi, V. M., Dib, R., Woods, P. M., Gavriil, F. P., \& Archibald, A. 2008, ApJ, 686, 520 